\title{
Asymmetric Injection in Organic Transistors via Direct SAM Functionalization of Source and Drain Electrodes
}

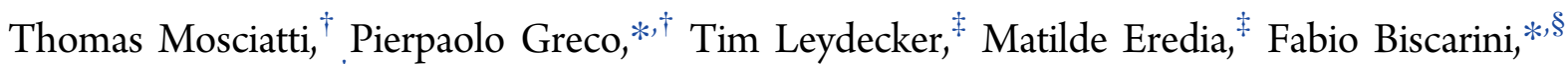
and Paolo Samori* ${ }^{*} \$$

\author{
${ }^{\dagger}$ Scriba Nanotecnologie, via di Corticella 183/8, 40128 Bologna, Italy \\ ${ }^{\ddagger}$ University of Strasbourg, CNRS, ISIS UMR 7006, 8 allée Gaspard Monge, F-67000 Strasbourg, France \\ ${ }^{\S}$ Life Science Department, Università di Modena e Reggio Emilia, Via Campi 103, 41125 Modena, Italy
}

Supporting Information

\begin{abstract}
The fabrication of organic optoelectronic devices integrating asymmetric electrodes enables optimal charge injection/extraction at each individual metal/semiconductor interface. This is key for applications in devices such as solar cells, light-emitting transistors, photodetectors, inverters, and sensors. Here, we describe a new method for the asymmetric functionalization of gold electrodes with different thiolated molecules as a viable route to obtain two electrodes with drastically different work function values. The process involves an ad hoc design of electrode geometry and the use of a polymeric mask to protect one electrode during the first functionalization step. Photoelectron yield ambient spectroscopy and X-ray photoelectron spectrometry were used to characterize the energetic properties and the composition of the asymmetrically functionalized electrodes. Finally, we used poly(3-hexylthiophene)-based organic thin-film transistors to show that the asymmetric electronic response stems from the different electronic structures of the functionalized electrodes.
\end{abstract}

\section{INTRODUCTION}

Surface functionalization is widely used in organic electronics for modulating interfacial properties at the micro- and nanoscale. ${ }^{1}$ Self-assembled monolayers (SAMs) of molecules with a thiol head group enable chemical and supramolecular functionalization of gold electrodes in organic thin-film transistors (OTFTs). ${ }^{2}$ Such functionalization of the $\mathrm{Au}$ electrodes is of pivotal importance for controlling the charge injection at the organic-semiconductor interfaces, ${ }^{3}$ balancing hole and electron injection, thus lowering the interfacial resistance at contacts. ${ }^{4}$ Furthermore, the possibility of grafting specific functional units on Au electrodes is key for applications in (bio)sensing and multifunctional devices. ${ }^{5}$ An SAM induces a shift of the Fermi level by chemisorption and introduces a dipole facing the metal surface, each contributing to an increase or decrease of the metal work function (WF) depending on the sign of the shift and the dipole orientation, respectively. ${ }^{6}$ As a consequence, it is possible to tune the WF of an electrode to match the highest occupied molecular orbital (HOMO) (lowest unoccupied molecular orbital -LUMO) of a p-type (n-type) semiconductor, thus enabling the fabrication of devices with ohmic contacts at the metal-semiconductor interfaces. ${ }^{7}$ The direction of the WF shift depends on the nature of the substituent group inserted at the $\omega$-position of the thiolated molecule. ${ }^{8}$ Alkanethiols are known to lower the WF, whereas halogenated terminated thiols are known to increase it. 9 The insertion of an SAM at the metal-semiconductor interface also has more subtle effects on the charge injection, distinct from the WF shift. In particular: (i) it may affect the wettability of the electrode and the packing motif that the organic semiconductor adopts on surfaces and in the bulk, ${ }^{10}$ and (ii) it introduces a nonconductive barrier that must be overcome or crossed by the charge carriers to be injected from the electrode into the semiconductor. ${ }^{11}$ The tunneling resistance increases exponentially with the length of the thiolated molecule, whereas it decreases with their degree of conjugation. $^{2 \mathrm{~b}}$ In addition, the molecular details of the moieties forming the SAM bring into play on some finer aspects, such as the emergence of odd-even effects in the effective charge mobility versus chain length. ${ }^{12}$ During the last decade, the research endeavor has been mostly focused on the symmetric functionalization of the source-drain electrodes, that is, with one molecular function exposed equally on the surface of the electrodes. In 2010, Liscio and co-workers have proposed a bottom-up technique for achieving asymmetric electrode functionalization. ${ }^{13}$ It consisted of a four-step procedure, which started with the symmetric SAM growth on both electrodes, followed by the electrochemical desorption of the

Received: May 28, 2017

Accepted: June 29, 2017

Published: July 12, 2017 
(a)

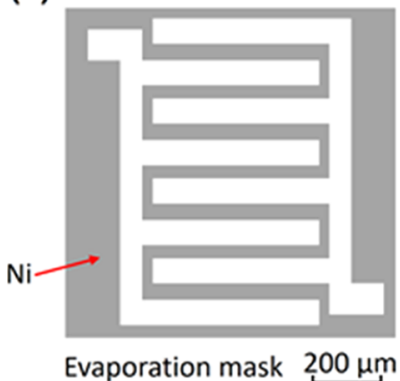

(b)

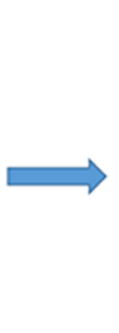

(f)

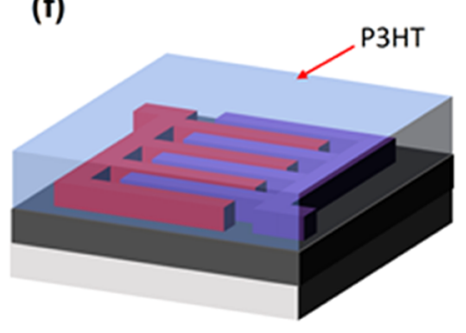

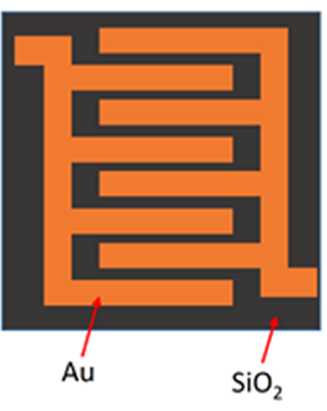

(c)

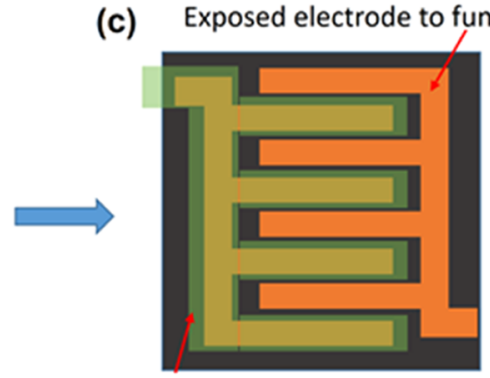

Polymeric Mask

rode (d)

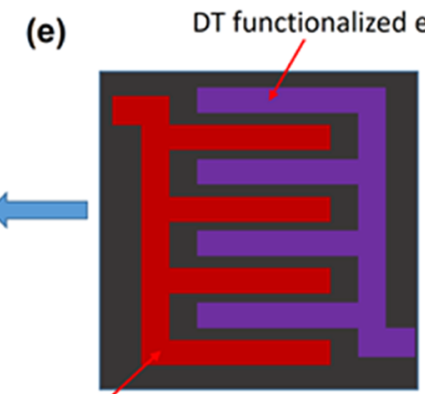

PFDT functionalized electrode

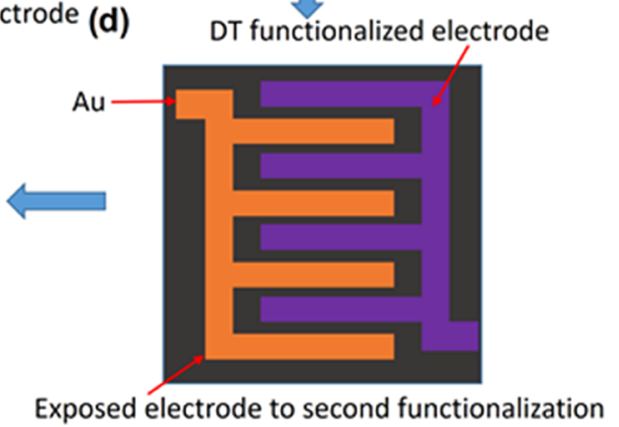

Figure 1. Fabrication procedure of the asymmetric electrode and OTFT. (a) Evaporation mask is cut on a nickel plate. (b) Mask is used to evaporate the electrode on $\mathrm{SiO}_{2}$ substrate. (c) Protective mask is placed on top of one electrode. (d) Electrode with the protective mask is placed in the solution and the nonprotected electrode is functionalized with DT. The polymeric mask is then removed by mechanical peeling. (e) Sample is placed in the PFDT solution to functionalize the second electrode with the fluorinated thiol. Finally, (f) poly(3-hexylthiophene) (P3HT) is spin-coated on top to finalize a bottom-gate, bottom-contact asymmetric OTFT.

SAM from only one of the two electrodes, followed by the chemisorption of a second thiolated molecule on the previously uncoated electrode. Asymmetric electrodes have already a wide range of applications. They are currently developed by employing different metals and are successfully used to enhance extraction of holes and electrons for application in solar cells, ${ }^{14}$ light-emitting transistors (LETs), inverters, photodetectors, and sensors. ${ }^{15}$ However, the molecular approach, via the asymmetric functionalization, presents the distinctive advantage of using electrodes of the same metal ( $\mathrm{Au}$ in this case), thus making the fabrication easier and quicker. Being based on the broad arsenal of thiolated molecules available possessing distinct characteristics, it also represents a versatile solution. Moreover, such specific functionalization allows one to control the interfacial properties, like wettability and the tunneling barrier of each electrode, thus enabling the tuning of the properties of each individual electrode. Unfortunately, the fabrication method proposed by Liscio et al. has some drawbacks. It involves the use of strong alkaline solutions that enhance bias stress during operations and may lead to the delamination of the electrodes from the substrate during the SAM desorption step. Because of these difficulties, asymmetric electrodes via SAM chemisorption of different thiols have not been proposed in the literature for a working device. More importantly, a fundamental piece in the field of nanotechnology applications, that is, a working asymmetric functionalized OFET, has not been reported to date.

\section{RESULTS AND DISCUSSION}

In this work, we propose a robust and reliable approach to the asymmetric functionalization via SAM of interdigitated electrodes by exploiting different microfabrication techniques. The process relies on the ad hoc design of the electrode shape and geometry. In this way, it is possible to fabricate a protective mask to cover just one of the two electrodes during the first SAM functionalization step. It requires precise microcutting and microalignment of the substrates but does not involve any step of photolithography. The proposed procedure is simple, is promptly implemented in any laboratory, and does not require further (electro)chemical desorption procedure.

For our purpose, we have chosen two thiolated molecules forming SAMs that induce a substantial energy asymmetry between the $\mathrm{Au}$ electrodes on which they chemisorb: decanethiol (DT) and $1 \mathrm{H}, 1 \mathrm{H}, 2 \mathrm{H}, 2 \mathrm{H}$-perfluorodecanethiol (PFDT). Their SAMs lead to the change of the WF of the electrodes in opposite directions due to the surface dipole moments that arise from the opposite polarity of the linear chains: the alkyl chain is weakly positively polar, with respect to thiol head, whereas the halogenated chain is strongly negatively polarized with respect to the thiol head. This yields a strong asymmetry of the charge injection in the target device. Because the SAM thickness is the same (differing less than $0.1 \mathrm{~nm}$ ), ${ }^{16}$ the tunneling barrier will have the roughly the same geometrical length scale at both electrodes. Tunneling resistance is characterized by the exponential inverse decay length, $\beta$, of the tunneling probability. The reported $\beta$ values for fluorinated chains are slightly larger than those for alkyl chains. ${ }^{17}$ In the present work, we will show that despite the difference in $\beta$ values, the asymmetry in the electrical properties of the OTFTs can be ascribed mostly to the change in WF at the zero-th order, with higher-order contributions from other electronic effects (energy of the localized states assisting through-bond tunneling, polarization effects, and vibrations).

For this work, we have designed a contact mask for patterning interdigitated electrodes by thermal evaporation on target substrates. The masks were fabricated using laser scan 

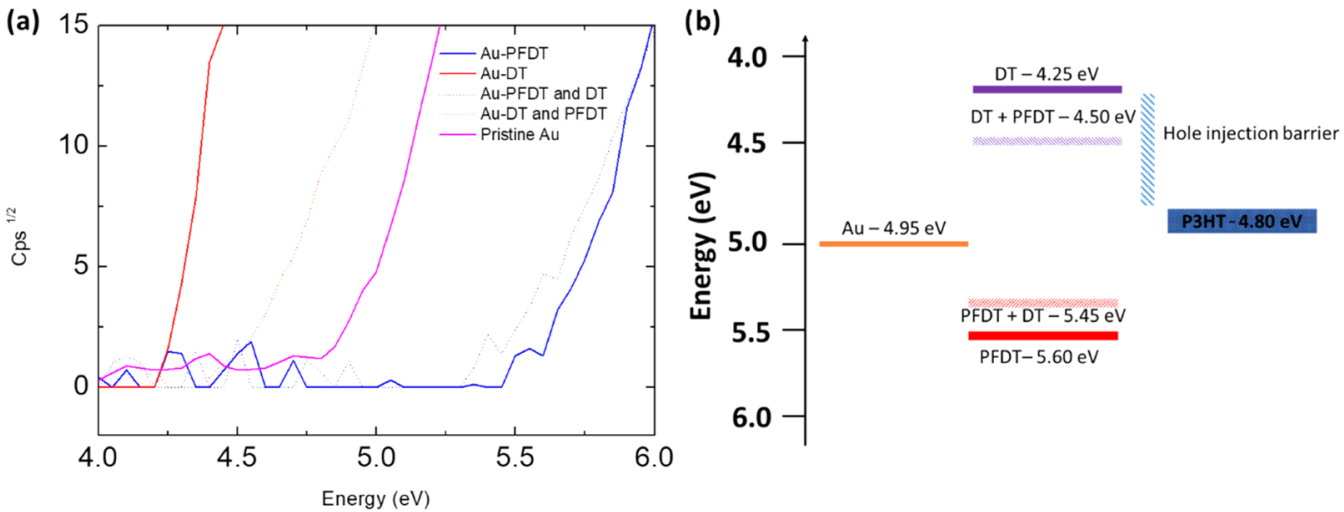

Figure 2. (a) Photoelectron yield spectra of SAM of PFDT on gold (solid line-blue), SAM of PFDT on gold after immersion in DT solution (dot line, royal blue), SAM of DT on gold (solid line-red), and SAM of DT on gold after immersion in PFDT solution (dot line-dark red). Photoelectron yield spectrum of pristine gold is reported in violet. (b) Energy levels extracted from (a) of the studied system. Meshed levels indicate the values measured on devices upon the second functionalization step. P3HT HOMO level and gold WF are also reported.

lithography. The laser scan marker Marko (Laserpoint SRL, Milan, Italy) has a pulsed ( $10 \mathrm{ps}$ width, $20 \mathrm{kHz}$ repetition rate, $50 \%$ duty cycle) Nd:YAG infrared (IR) laser centered at $\lambda=$ $1064 \mathrm{~nm}$. Nickel plates, $1 \mathrm{~mm}$ thick, were cut with the laser, while freestanding on the Marko's stage. A schematic drawing of the masks is shown in Figure 1a. They were designed with a channel width $(W)$ of $150 \mathrm{~mm}$ and a channel length $(L)$ of 500 $\mu \mathrm{m}$, yielding a $W / L=300$. A typical functionalization and fabrication procedure starts by employing the evaporator masks to evaporate onto the $\mathrm{n}++-\mathrm{Si} / \mathrm{SiO}_{2}$ substrates first a $5 \mathrm{~nm}$ thick $\mathrm{Cr}$ adhesion layer and then a $45 \mathrm{~nm}$ thick gold film (Figure $1 \mathrm{~b}$ ).

A polymer mask was designed with the aim of completely protecting one of the two electrodes. The selected material for the polymeric mask fabrication is a laminated multilayer (poly(ethylene terephthalate)/acrylic), which guarantees a perfect adhesion and avoids leakage of the solution over the protected electrodes. The mask was laser-cut with Marko starting from the drawing of the evaporation mask. The protective mask was planned to perfectly cover the electrode with a margin of $250 \mu \mathrm{m}$ on each side. The electrodes were ozone cleaned, and the mask was optically aligned on top of one of the electrodes (Figure 1c). The protective mask avoids the solution to enter in contact with the electrode. Such protective masks are not reusable, but their cost can be estimated at about $0.01 €$. The sample was immersed for $36 \mathrm{~h}$ in a $1 \mathrm{mM}$ solution of DT in EtOH to grow the SAM on electrode 1. The sample was cleaned with warm $\mathrm{EtOH}, \mathrm{CHCl}_{3}$, and 2propanol. The polymer mask was then removed by mechanically peeling it off with tweezers (Figure 1d), and the sample was cleaned again with 2-propanol. After drying under $\mathrm{N}_{2}$, the sample was put for $36 \mathrm{~h}$ in a $1 \mathrm{mM}$ solution of PFDT in $\mathrm{EtOH}$ to functionalize electrode 2 (Figure 1e). To rinse all physisorbed thiolated molecules, the sample was cleaned by successively squirting warm $\mathrm{EtOH}, \mathrm{CHCl}_{3}$, and 2-propanol and drying under $\mathrm{N}_{2}$ flow. To ensure complete removal of eventual mask residues, the sample was sonicated in toluene for $10 \mathrm{~min}$ at $60{ }^{\circ} \mathrm{C}$.

The decision of functionalizing first with DT and then with PFDT was taken after analyzing the effect on the final WF of gold. Thiols with longer chains typically exhibit greater affinity for gold with respect to shorter ones, and usually this criterion is applied to decide which thiol should be used first. ${ }^{13}$ In our case, having the two thiols of the same length, we performed photoelectron yield ambient (PEYA) spectroscopy measure- ments to gain insight into the substitution process occurring at the electrode surface. A surface of $1 \mathrm{~cm}^{2}$ of $\mathrm{Au}$ has been functionalized with the procedure described above. In Figure 2a are reported the spectra recorded with the PEYA technique. A difference exceeding $1.2 \mathrm{eV}$ is obtained by functionalizing $\mathrm{Au}$ with DT and PFDT. Such marked difference is expected to yield a strong asymmetry in the OTFTs. When DT was employed in the first step, we measured the WF of the SAMfunctionalized electrode as being $4.25 \pm 0.05 \mathrm{eV}$. Such a low value is in good agreement with previous studies. ${ }^{18}$ In contrast, $\mathrm{Au}$ functionalized first with PFDT exhibited an effective WF value of $5.60 \pm 0.05 \mathrm{eV}$, the higher value being expected from perfluoro-methyl terminated moieties. ${ }^{4 a}$ Those samples were then immersed into EtOH solutions of PFDT and DT, respectively, as second functionalization, and their effective WF was measured again. In dotted lines (Figure 2a) are reported the spectra obtained after an already functionalized gold surface was placed in a solution of the other thiols. A slight shift is observed in both cases. This can be due to a partial exchange reaction between the thiols occurring at the interface and to the second thiol filling eventual defects occurring during the first SAM functionalization step. The sample first functionalized with DT and then PFDT presented an effective WF of $4.50 \pm$ $0.05 \mathrm{eV}$, which implies a downward shift of $0.25 \mathrm{eV}$ of the surface vacuum level. The sample first functionalized with PFDT and then DT exhibited a WF value of $5.40 \pm 0.05 \mathrm{eV}$, with a shift of $0.15 \mathrm{eV}$ upward of the surface vacuum level. These shifts are due to ligand exchange at the electrodes occurring during the second functionalization, when the protective mask is removed. Pristine gold showed a WF value of $4.95 \pm 0.05 \mathrm{eV}$ (see the Supporting Information for complete details). Our target device was based on an active layer of P3HT. When spin-coated from chloroform, the HOMO level of P3HT is centered at around $4.8 \mathrm{eV} .^{19}$ The energy levels of our system are depicted in Figure $2 \mathrm{~b}$. Hence, at a first glance, the DT-functionalized electrode will present, with respect to $\mathrm{P} 3 \mathrm{HT}$, an injection barrier for holes due to the lower WF, whereas for the PFDT-functionalized electrode no injection barrier should occur. Electrodes first functionalizing with PFDT and then DT will produce an asymmetry of (1.15 \pm $0.05)$, whereas proceeding the other way around would lead to a $(1.10 \pm 0.05)$ asymmetry. All of the extracted data are summarized in Table S1. It is evident that any order of functionalization would lead to the same outcome (dashed 

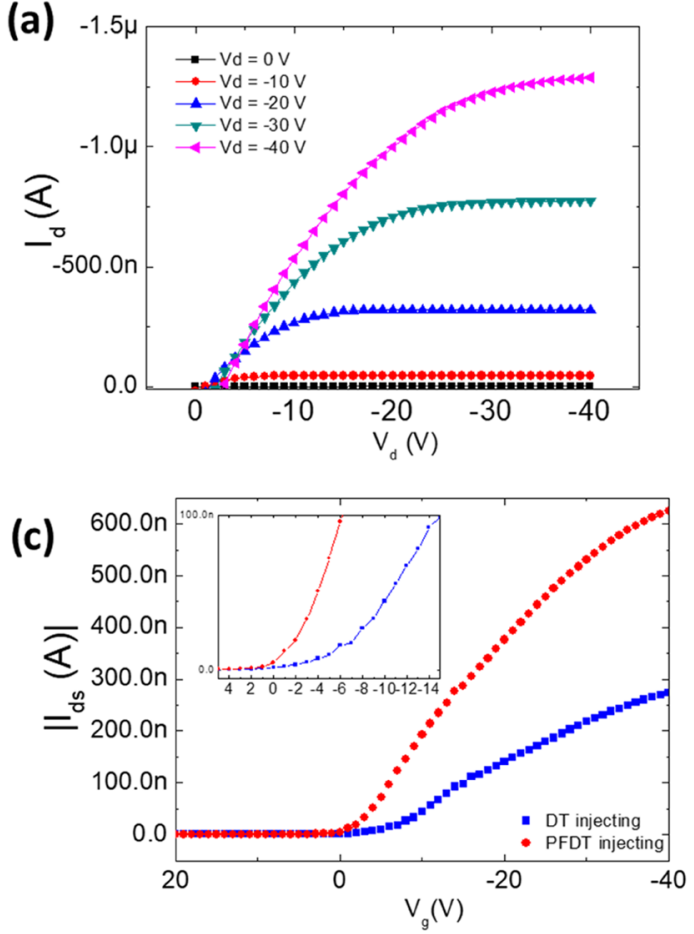

(b)

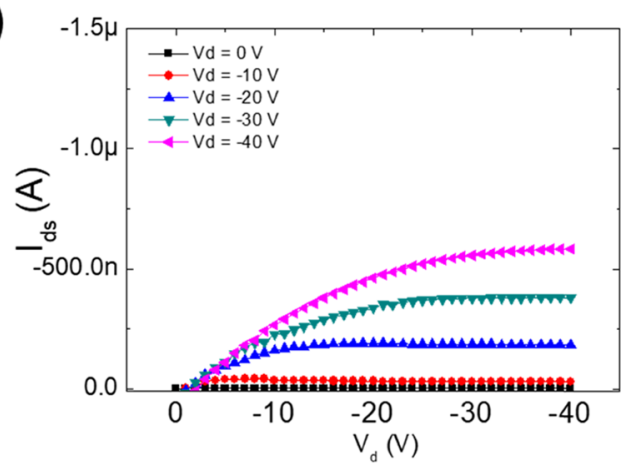

(d)

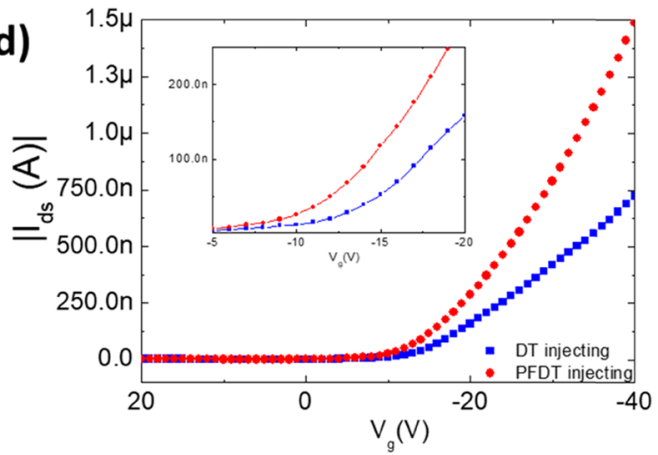

Figure 3. Output characteristic of the OTFT device when injecting from (a) the PFDT-functionalized electrode and (b) the DT-functionalized electrode. $I_{\mathrm{d}}-V_{\mathrm{d}}$ curves are on the same scale so that differences in the drain current between the two different configurations can be better highlighted. Transfer characteristics are reported with $I_{\mathrm{ds}}$ in the llin scale (c) at $V_{\mathrm{ds}}=0.3 \mathrm{~V}$ and (d) at $V_{\mathrm{ds}}=0.7 \mathrm{~V}$. Curves obtained when DT is injecting are reported in blue and those obtained when PFDT is injecting are reported in red. In the inset, the area close to $V_{\text {th }}$ is reported magnified to appreciate better the threshold voltage shift.

levels in Figure 2b), that is, an electrode with a lower WF with respect to the HOMO of $\mathrm{P} 3 \mathrm{HT}$ and the other one with a higher WF value. Therefore, a different electronic response should be observed when injecting from the DT-functionalized electrode (higher injection barrier for hole carriers, else lower injection barrier for electron carriers) or from the PFDTfunctionalized electrode (no injection barrier for hole carriers, else higher injection barrier for electron carriers).

As aforementioned, we started with DT first. This choice has been made to evaluate the different functionalization of the interdigitated electrode by X-ray photoelectron spectroscopy (XPS) through the survey of C-F and F signals. XPS spectra have been recorded in the six central fingers of the device, that is, three belonging to electrode 1 and three belonging to electrode 2. As displayed in Figure S2, the $\mathrm{S}-\mathrm{Au}$ bond is present in each of them, confirming the successful functionalization of the electrodes via chemisorption. The $\mathrm{C}-\mathrm{F}$ signal is relatively stronger in the fingers not covered by the mask, with respect to the ones protected by the mask. This confirms the successful asymmetric functionalization of the electrodes. The small amount of $\mathrm{F}$ signal in the even electrodes should be ascribed to a partial exchange between DT and PFDT, as discussed previously in the PEYA measurements (see the Supporting Information for details).

Once having verified the proper asymmetrical functionalization of the electrodes, we fabricated OTFTs using P3HT as a semiconductor. A $5 \mathrm{mg} \mathrm{mL}^{-1} \mathrm{P} 3 \mathrm{HT}$ solution in chloroform was spin-coated over the electrodes in a nitrogen atmosphere. We obtained a homogenous film completely covering the electrodes' area (see the Supporting Information). The sample was annealed at $50{ }^{\circ} \mathrm{C}$ for $2 \mathrm{~h}$ to ensure complete evaporation of the solvents. Such low-temperature annealing is of fundamental importance to preserve the functionalization of the electrodes. ${ }^{20}$

We have tested our devices under a nitrogen atmosphere to avoid the occurrence of doping of $\mathrm{P} 3 \mathrm{HT}$. The devices were tested first connecting one electrode to the source and then the other one. The results of the electrical characterization are reported in Figure 3. We started by keeping the DT electrode grounded and therefore the polarization was applied to the PFDT-functionalized electrode. In this configuration, the charges are injected from the PFDT electrode and collected by the DT electrodes in a stepwise configuration. ${ }^{21}$ The output curves (Figure 3a) show a perfect separation between each different gate polarization. A small S-shape is observed close to the $0 \mathrm{~V}$ drain voltage, indicating the presence of a small contact resistance in the device. ${ }^{10}$ Such a shape has already been reported in the literature for thin-film transistors based on source and drain electrodes functionalized with chemisorbed monolayers of thiolated molecules. ${ }^{3 a, 22}$ It can be attributed to the fact that we introduced a barrier at the interface that must be overcome by tunneling.

Figure $3 b$ portrays the output characteristic recorded with inverted connections, that is, with the charges injected from the DT electrode (source) and collected by the PFDT one (drain). A lower current is observed together with an imperfect spacing of the curves. Again, a small S-shape close to $0 \mathrm{~V}$ drain voltage is present. We ascribe the lowered current to a less favorable injection of holes, due to the low-injecting WF of the DTcoated electrode, which leads to an energy barrier that must be overcome by the injected holes. Transfer characteristics are reported in Figure 3c,d at linear (high $V_{\mathrm{ds}}=-10 \mathrm{~V}$ ) and saturation (low $V_{\mathrm{ds}}=-40 \mathrm{~V}$ ) regimes, respectively. In both cases, a lower current is observed when injecting from the DT 
Table 1. Linear and Saturation $\mu$ and $V_{\text {th }}$ Extracted from Transfer Curves for DT and PFDT Injecting Electrodes of Representative Asymmetric OTFT ${ }^{a}$

$\begin{array}{lcccc} & \text { linear regime mobility }\left(\mathrm{cm}^{2} \mathrm{~V}^{-1} \mathrm{~s}^{-1}\right) & V_{\text {th }} \text { linear regime }(\mathrm{V}) & \text { saturation regime mobility }\left(\mathrm{cm}^{2} \mathrm{~V}^{-1} \mathrm{~s}^{-1}\right) & V_{\text {th }} \text { saturation regime }(\mathrm{V}) \\ \text { DT injecting } & (7.2) \times 10^{-3} & (-2.6) & (8.9) \times 10^{-3} & (-1.6) \\ \text { PFDT injecting } & (8.1) \times 10^{-3} & (1.2) & (12.3) \times 10^{-3} & (0.8) \\ \Delta & (1.4 \pm 0.7) \times 10^{-3} & (3.5 \pm 1.1) & (3.0 \pm 0.3) \times 10^{-3} & (1.9 \pm 1.0)\end{array}$

${ }^{a}$ In the last row, the $\left(\mu_{\mathrm{PFDT}}-\mu_{\mathrm{DT}}\right.$ and $\left.V_{\mathrm{th}, \mathrm{PFDT}}-V_{\mathrm{th}, \mathrm{DT}}\right)$ average difference calculated on at least six samples in linear and saturation regimes are reported.

electrode (blue) together with a small shift toward more negative values of the threshold voltage $\left(V_{\mathrm{th}}\right.$, highlighted in the insets). The negative shift of $V_{\text {th }}$ indicates that the DTfunctionalized electrode effectively acts as a trap for carriers. Thus, a higher (in magnitude) voltage is needed for charges to be injected into the semiconducting active layer, due to the increased energy barrier. In addition, it is possible to notice in the positive part of the transfer curve that the current is larger when injecting from DT electrodes, as expected from the energy level alignment at the interface. In Table 1 are reported the extracted $V_{\text {th }}$ and mobility values when injecting from DT and PFDT, respectively, and the difference in the values $\left(\mu_{\mathrm{PFDT}}\right.$ $-\mu_{\mathrm{DT}}$ and $\left.V_{\mathrm{th}, \mathrm{PFDT}}-V_{\mathrm{th}, \mathrm{DT}}\right)$ between the two configurations averaged on over six samples.

The difference between mobility values in the two configurations is about 30\% higher for injecting from PFDT, whereas the threshold voltage shift is $2-3 \mathrm{~V}$ toward more negative values for injecting from DT. It must be noticed that all the devices injecting from PFDT always yielded higher hole mobilities compared to those injecting from DT. Moreover, $V_{\text {th }}$ was always more positive with injection from the PFDT/Au electrode. This demonstrates a perfect reproducibility of the asymmetric transistors. In addition, leakage current was always at least 1 order of magnitude lower than the source-drain current (Figure S9), proving the robust approach to the device fabrication employed.

Such differences, albeit being significant, are not as marked as one could expect. To rationalize the results, we consider the total resistance, $R_{\mathrm{TOT}}$, of the transistor as given by

$$
R_{\mathrm{TOT}}=R_{\mathrm{ch}}+R_{\mathrm{i}}
$$

$R_{\mathrm{ch}}$ is the resistance of the channel and $R_{\mathrm{i}}$ is the interface or contact resistance. When the channel length is short, usually below 10-20 $\mu \mathrm{m}, R_{\mathrm{i}}$ becomes dominant in devices with bare $\mathrm{Au}$ electrodes. ${ }^{23}$ For longer channels, $R_{\mathrm{ch}}$ becomes increasingly more important. However, our results show that even with a channel length of $500 \mu \mathrm{m}$, the interfacial resistance, $R_{\mathrm{i}}$, is sizable with our functionalization. Then, we discuss the factors that may justify our observation. Wetting of the channel material on electrodes may play a role. Surfaces with a lower surface energy and matching that of P3HT lead to better packing, favorable orientation, and less energy disorder ${ }^{10}$ of the semiconductor at the interface. ${ }^{18 a}$ In the present case, the DT-functionalized electrode exhibits a water contact angle of $105.5 \pm 0.7^{\circ}$, whereas the PFDT-coated one is $103.5 \pm 0.7^{\circ}$ (see Figure S8 for data on extracted contact angle). We estimated that the two functionalized surfaces exhibit very close surface free energy values (see the Supporting Information). This strongly hints that surface tension should not significantly affect the morphology of the P3HT film at the two electrodes. This is in agreement with the homogenous film we obtain also on the electrodes (see the Supporting Information). Hence, we believe that surface energy cannot justify the experimental difference observed. As previously discussed, the larger inverse decay length, $\beta$, of the fluorinated chains might partially mitigate the enhancement effects due to the more favorable energy alignment between HOMO of P3HT and the Fermi level in the case of PFDT electrodes. This may partially compensate the WF difference leading to the significant, albeit not extraordinary in magnitude, mismatch between the two different injection schemes. In addition, the presence of the SAM at the interface can affect the electrical output. The monolayer is electrostatic and chemically bound at the surface so there is a thiol-gold dipole and a dipole due to the head group of the monolayer. $^{2 \mathrm{~b}, 24}$ This can generate low-lying trap states at the $\mathrm{Au} / \mathrm{SAM} / \mathrm{P} 3 \mathrm{HT}$ interface ${ }^{\mathrm{Ib}}$ as in the case of PFDT electrodes, which can also partially hinder the asymmetric outcome of the device. For the sake of completeness, we have performed all possible control experiments. The pristine $\mathrm{Au}$ electrode presented better performances due to the ohmic contact between the bare metal and P3HT. The observed response is symmetrical to the reversal of injection by inverting the source grounding. Devices with the electrodes both functionalized with the same molecules, being either DT or PFDT, were also prepared. In this case, a large shift toward negative values of $V_{\text {th }}$ was observed for devices bearing DT molecules on the electrodes. This is expected due to the large injection barrier induced by SAM. All those configurations presented no asymmetry when the ground source was swapped (see Figures S4 and S5). Finally, partially functionalized electrodes, that is, just one electrode coated with a chemisorbed SAM, have been prepared. Here, a similar behavior to the asymmetric configuration performance was measured. Devices with a DT electrode and a bare gold electrode showed a similar performance as the previously discussed asymmetric DT/ PFDT devices. This finding is in agreement with the energetic alignment at the interface reported in Figure 2 because the gold has an ohmic contact with P3HT, whereas the DT electrode gives rise to an energy barrier. This result paves the way for a smart level alignment, with just one electrode functionalized without the need of an electrochemical process. The other asymmetric devices, with one electrode coated with PFDT and the other one with bare gold, also showed a small asymmetry. In particular, when injecting from gold, a $15 \%$ increase in mobility is observed. Such a difference can be attributed to the fact that on the $\mathrm{Au}$ electrode the contribution to the interfacial resistance is smaller. All of the curves and extracted values are reported in Figures S6 and S7. The results from the control experiments, together with the ones discussed here, support the fact that the mask strategy is effective for the asymmetric functionalization of electrodes. They also show that with the fine tuning of single electrodes it is possible to obtain asymmetric electrical behavior even with just one electrode functionalized. 


\section{CONCLUSIONS}

In conclusion, we have devised a new method for the asymmetric functionalization of gold electrodes with two different thiolated molecules for applications in optoelectronic devices. Our technique is robust and does not involve any electrochemical process. It made possible to individually tune the WF of the source and drain electrodes, yielding a significant asymmetry in the electrical characteristics of the tested OTFTs depending on the polarization of the source and drain. Recent improvement in laser processing made it possible to scale down patterning resolution to the $100 \mathrm{~nm}$ scale. ${ }^{25}$ Such innovation paves the route toward the design of masks and their alignment with such a spatial resolution, ready for device integration. In a broader view, having an electrode with a favorable injection for holes and the other one for electrons sets the grounds for a platform to further develop more complex electronic devices and functions. Our method opens the possibility to enhance the response of large-area device architectures through interfacial tailoring of single electrodes, for applications in LET, OTFTs, inverters, solar cells, and sensors.

\section{MATERIALS AND METHODS}

Mask for the Evaporation Fabrication Procedure. A nickel plate was used to fabricate the evaporation mask. The mask was cut with the laser marker Marko. It has a short-pulsed Nd:YAG IR laser centered at $\lambda=1064 \mathrm{~nm}$. Typical cutting parameters were a laser power of $8 \mathrm{~W}$ and a frequency of 20 $\mathrm{kHz}$. The nickel plate was put freestanding on the Marko's stage using four magnets (Figure S1). The stage was moved by the software, which can move it in the $X-Y$ directions. The focus was controlled by moving the laser head in the $Z$ direction. The movement was controlled with an accuracy of less than $1 \mu \mathrm{m}$ in the $X-Y-Z$ directions. The laser beam was moved over the surface according to the design input to the software. The optimal cutting speed was found to be $20 \mu \mathrm{m} \mathrm{s}^{-1}$. A total of 25 passages were needed to achieve a complete cut of the metal plate. A 10 min timescale was necessary to obtain the mask. The mask was then polished with a polish paste and cleaned with water and soap. Finally, a sonication in ethanol for $10 \mathrm{~min}$ was performed to ensure complete removal of all the remaining metallic powder and polish paste.

Protection Mask for the Electrode Fabrication Procedure. A polymeric laminated film with glue on one side was purchased from 3M (product ID 3M-UU003122981). The polymer was put stretched on the Marko's stage and cut with a power of $3 \mathrm{~W}, 20 \mathrm{kHz}$ at $20 \mu \mathrm{m} \mathrm{s}^{-1}$. All other details have been described in the previous paragraph. Two scans were needed to obtain the cut, in a process lasting $2 \mathrm{~s}$ for each mask.

Electrode Fabrication and Functionalization. First, 5 $\mathrm{nm}$ of $\mathrm{Cr}$ and then $45 \mathrm{~nm}$ of gold were evaporated through the evaporation mask on a $\mathrm{n}++-\mathrm{Si} / \mathrm{SiO}_{2}$ substrate (Fraunhofer IPMS). They consisted of $\mathrm{n}++-\mathrm{Si}$ substrates with $230 \mathrm{~nm}$ of thermally grown $\mathrm{SiO}_{2}$ as the gate dielectric ( $15 \mathrm{nF}$ capacitance). The doped silicon layer can act as a gate electrode, whereas the silicon oxide layer can act as a dielectric. They were ozone cleaned for $5 \mathrm{~min}$, followed by $25 \mathrm{~min}$ of incubation. The protective mask was placed over one of the two electrodes covering it completely. The mask was manually placed on it, with the help of a long focal microscope. In a cylindrical weighing bottle, $3 \mu \mathrm{L}$ of DT (Sigma) was dissolved in $20 \mathrm{~mL}$ of EtOH. Then, the sample was placed in the solution. After $36 \mathrm{~h}$, it was cleaned with warm ethanol, chloroform, and 2-propanol.
The mask was then removed, and the sample was cleaned again with 2-propanol. In another cylindrical weighing bottle, $5 \mathrm{mg}$ of PFDT (Sigma) was dissolved in $10 \mathrm{~mL}$ of EtOH. The sample without any protective mask was placed in the solution for $36 \mathrm{~h}$. The sample was then cleaned by squirting warm ethanol, chloroform, and 2-propanol. To ensure complete removal of the mask residues, the sample was sonicated in toluene for 10 $\min$ at $60{ }^{\circ} \mathrm{C}$. The final sample was then cleaned with 2propanol, followed by drying under nitrogen flow.

OTFT Fabrication. A $5 \mathrm{mg} \mathrm{mL}^{-1}$ solution of P3HT in chloroform was prepared and continuously stirred for $1 \mathrm{~h}$ at 35 ${ }^{\circ} \mathrm{C}$ to ensure complete solubilization. A volume of $150 \mu \mathrm{L}$ of the solution was spin-coated ( $3000 \mathrm{rpm}, 1500 \mathrm{rpm} \mathrm{s}^{-2}, 60 \mathrm{~s}$ ) on the samples and annealed at $50{ }^{\circ} \mathrm{C}$ for $2 \mathrm{~h}$.

\section{ASSOCIATED CONTENT}

\section{Supporting Information}

The Supporting Information is available free of charge on the ACS Publications website at DOI: 10.1021/acsomega.7b00690.

Materials and methods; parameters extraction; XPS; WF measurements; pristine gold P3HT-based OTFTs; symmetric functionalized P3HT-based OTFTs; one electrode functionalized P3HT-based OTFTs; contact angle and surface energy measurements; leakage current; P3HT film on electrodes (PDF)

\section{AUTHOR INFORMATION}

\section{Corresponding Authors}

*E-mail: pgreco@scriba-nanotec.com (P.G.).

*E-mail: fabio.biscarini@unimore.it (F.B.).

*E-mail: samori@unistra.fr (P.S.).

ORCID $\odot$

Paolo Samorì: 0000-0001-6256-8281

\section{Author Contributions}

All authors have given approval to the final version of the manuscript.

Notes

The authors declare no competing financial interest.

\section{ACKNOWLEDGMENTS}

We would like to thank Dr. Oliver Fenwick for useful discussions. This work was financially supported by the EC through the Marie Curie ETN project iSwitch (GA No. 642196), the Labex projects CSC (ANR-10-LABX-0026 CSC) within the Investissement d'Avenir program ANR-10-IDEX0002-02, and the International Center for Frontier Research in Chemistry (icFRC).

\section{REFERENCES}

(1) (a) Love, J. C.; Estroff, L. A.; Kriebel, J. K.; Nuzzo, R. G.; Whitesides, G. M. Self-Assembled Monolayers of Thiolates on Metals as a Form of Nanotechnology. Chem. Rev. 2005, 105, 1103-1170. (b) Mosciatti, T.; Orgiu, E.; Raimondo, C.; Samorì, P. The role of size and coating in $\mathrm{Au}$ nanoparticles incorporated into bi-component polymeric thin-film transistors. Nanoscale 2014, 6, 5075-5080.

(2) (a) Casalini, S.; Bortolotti, C. A.; Leonardi, F.; Biscarini, F. Selfassembled monolayers in organic electronics. Chem. Soc. Rev. 2017, 46, 40-71. (b) Liu, C.; Xu, Y.; Noh, Y.-Y. Contact engineering in organic field-effect transistors. Mater. Today 2015, 18, 79-96.

(3) (a) Youn, J.; Dholakia, G. R.; Huang, H.; Hennek, J. W.; Facchetti, A.; Marks, T. J. Influence of Thiol Self-Assembled Monolayer Processing on Bottom-Contact Thin-Film Transistors 
Based on n-Type Organic Semiconductors. Adv. Funct. Mater. 2012, 22, 1856-1869. (b) Lee, J.; Park, J. S.; Lee, B.-L.; Park, J.-i.; Chung, J. W.; Lee, S. Ionic self-assembled monolayer for low contact resistance in inkjet-printed coplanar structure organic thin-film transistors. Org. Electron. 2014, 15, 2021-2026. (c) Fenwick, O.; Van Dyck, C.; Murugavel, K.; Cornil, D.; Reinders, F.; Haar, S.; Mayor, M.; Cornil, J.; Samorì, P. Modulating the charge injection in organic field-effect transistors: fluorinated oligophenyl self-assembled monolayers for high work function electrodes. J. Mater. Chem. C 2015, 3, 3007-3015.

(4) (a) Marmont, P.; Battaglini, N.; Lang, P.; Horowitz, G.; Hwang, J.; Kahn, A.; Amato, C.; Calas, P. Improving charge injection in organic thin-film transistors with thiol-based self-assembled monolayers. Org. Electron. 2008, 9, 419-424. (b) Jäger, C. M.; Schmaltz, T.; Novak, M.; Khassanov, A.; Vorobiev, A.; Hennemann, M.; Krause, A.; Dietrich, H.; Zahn, D.; Hirsch, A.; Halik, M.; Clark, T. Improving the Charge Transport in Self-Assembled Monolayer Field-Effect Transistors: From Theory to Devices. J. Am. Chem. Soc. 2013, 135, 4893-4900.

(5) (a) Casalini, S.; Shehu, A.; Destri, S.; Porzio, W.; Pasini, M. C.; Vignali, F.; Borgatti, F.; Albonetti, C.; Leonardi, F.; Biscarini, F. Organic field-effect transistors as new paradigm for large-area molecular junctions. Org. Electron. 2012, 13, 789-795. (b) Mosciatti, T.; del Rosso, M. G.; Herder, M.; Frisch, J.; Koch, N.; Hecht, S.; Orgiu, E.; Samorì, P. Light-Modulation of the Charge Injection in a Polymer Thin-Film Transistor by Functionalizing the Electrodes with Bistable Photochromic Self-Assembled Monolayers. Adv. Mater. 2016, 28, 6606-6611. (c) Crivillers, N.; Orgiu, E.; Reinders, F.; Mayor, M.; Samorì, P. Optical Modulation of the Charge Injection in an Organic Field-Effect Transistor Based on Photochromic Self-AssembledMonolayer-Functionalized Electrodes. Adv. Mater. 2011, 23, 14471452. (d) Zhang, H.; Chen, H.; Ma, W.; Hui, J.; Meng, S.; Xu, W.; Zhu, D.; Guo, X. Photocontrol of charge injection/extraction at electrode/semiconductor interfaces for high-photoresponsivity organic transistors. J. Mater. Chem. C 2016, 4, 5289-5296.

(6) de Boer, B.; Hadipour, A.; Mandoc, M. M.; van Woudenbergh, T.; Blom, P. W. M. Tuning of Metal Work Functions with SelfAssembled Monolayers. Adv. Mater. 2005, 17, 621-625.

(7) (a) Brédas, J. L.; Calbert, J. P.; da Silva Filho, D. A.; Cornil, J. Organic semiconductors: A theoretical characterization of the basic parameters governing charge transport. Proc. Natl. Acad. Sci. U.S.A. 2002, 99, 5804-5809. (b) Hamadani, B. H.; Natelson, D. Temperature-dependent contact resistances in high-quality polymer field-effect transistors. Appl. Phys. Lett. 2004, 84, 443-445.

(8) (a) Cornil, D.; Cornil, J. Work-function modification of the (1 1 1) gold surface upon deposition of self-assembled monolayers based on alkanethiol derivatives. J. Electron Spectrosc. Relat. Phenom. 2013, 189, 32-38. (b) Yasutaka, K.; Masatoshi, K. Work function of gold surfaces modified using substituted benzenethiols: Reaction time dependence and thermal stability. Appl. Phys. Express 2014, 7, No. 035701. (c) Daniel, B.; Daniel, P.; Christian, E.; Robert, C.; Wolfgang, F.; Karsten, R.; Cornelius, G.; Martin, W. Mixed selfassembled monolayers of azobenzene photoswitches with trifluoromethyl and cyano end groups. J. Phys.: Condens. Matter 2012, 24, No. 394015.

(9) (a) Kim, H.; Meihui, Z.; Battaglini, N.; Lang, P.; Horowitz, G. Large enhancement of hole injection in pentacene by modification of gold with conjugated self-assembled monolayers. Org. Electron. 2013, 14, 2108-2113. (b) Rusu, P. C.; Brocks, G. Surface Dipoles and Work Functions of Alkylthiolates and Fluorinated Alkylthiolates on $\mathrm{Au}(111)$. J. Phys. Chem. B 2006, 110, 22628-22634.

(10) Orgiu, E.; Crivillers, N.; Rotzler, J.; Mayor, M.; Samorì, P. Tuning the charge injection of P3HT-based organic thin-film transistors through electrode functionalization with oligophenylene SAMs. J. Mater. Chem. 2010, 20, 10798-10800.

(11) Cheng, X.; Noh, Y.-Y.; Wang, J.; Tello, M.; Frisch, J.; Blum, R. P.; Vollmer, A.; Rabe, J. P.; Koch, N.; Sirringhaus, H. Controlling Electron and Hole Charge Injection in Ambipolar Organic Field-Effect Transistors by Self-Assembled Monolayers. Adv. Funct. Mater. 2009, 19, 2407-2415.
(12) Stoliar, P.; Kshirsagar, R.; Massi, M.; Annibale, P.; Albonetti, C.; de Leeuw, D. M.; Biscarini, F. Charge Injection Across Self-Assembly Monolayers in Organic Field-Effect Transistors: Odd-Even Effects. J. Am. Chem. Soc. 2007, 129, 6477-6484.

(13) Liscio, A.; Orgiu, E.; Mativetsky, J. M.; Palermo, V.; Samorì, P. Bottom-Up Fabricated Asymmetric Electrodes for Organic Electronics. Adv. Mater. 2010, 22, 5018-5023.

(14) Dong, W.; Guo, Y.; Guo, B.; Liu, H.; Li, H.; Liu, H. Photovoltaic properties of $\mathrm{BiFeO}_{3}$ thin film capacitors by using $\mathrm{Al}$-doped zinc oxide as top electrode. Mater. Lett. 2013, 91, 359-361.

(15) Ullah, M.; Tandy, K.; Li, J.; Shi, Z.; Burn, P. L.; Meredith, P.; Namdas, E. B. High-Mobility, Heterostructure Light-Emitting Transistors and Complementary Inverters. ACS Photonics 2014, 1, 954-959.

(16) Pflaum, J.; Bracco, G.; Schreiber, F.; Colorado, R., Jr.; Shmakova, O. E.; Lee, T. R.; Scoles, G.; Kahn, A. Structure and electronic properties of $\mathrm{CH}_{3}$ and $\mathrm{CF}_{3}$-terminated alkanethiol monolayers on $\mathrm{Au}\left(\begin{array}{lll}1 & 1 & 1\end{array}\right)$ : a scanning tunneling microscopy, surface X-ray and helium scattering study. Surf. Sci. 2002, 498, 89-104.

(17) Liao, K.-C.; Bowers, C. M.; Yoon, H. J.; Whitesides, G. M. Fluorination, and Tunneling across Molecular Junctions. J. Am. Chem. Soc. 2015, 137, 3852-3858.

(18) (a) Leydecker, T.; Trong Duong, D.; Salleo, A.; Orgiu, E.; Samorì, P. Solution-Processed Field-Effect Transistors Based on Dihexylquaterthiophene Films with Performances Exceeding Those of Vacuum-Sublimed Films. ACS Appl. Mater. Interfaces 2014, 6, 21248-21255. (b) Singh, K. A.; Nelson, T. L.; Belot, J. A.; Young, T. M.; Dhumal, N. R.; Kowalewski, T.; McCullough, R. D.; Nachimuthu, P.; Thevuthasan, S.; Porter, L. M. Effect of Self-Assembled Monolayers on Charge Injection and Transport in Poly(3-hexylthiophene)-Based Field-Effect Transistors at Different Channel Length Scales. ACS Appl. Mater. Interfaces 2011, 3, 2973-2978.

(19) Orgiu, E.; Crivillers, N.; Herder, M.; Grubert, L.; Pätzel, M.; Frisch, J.; Pavlica, E.; Duong, D. T.; Bratina, G.; Salleo, A.; Koch, N.; Hecht, S.; Samorì, P. Optically switchable transistor via energy-level phototuning in a bicomponent organic semiconductor. Nat. Chem. 2012, 4, 675-679.

(20) Asadi, K.; Gholamrezaie, F.; Smits, E. C. P.; Blom, P. W. M.; Boer, B. d. Manipulation of charge carrier injection into organic fieldeffect transistors by self-assembled monolayers of alkanethiols. J. Mater. Chem. 2007, 17, 1947-1953.

(21) Tang, Q.; Li, H.; Liu, Y.; Hu, W. High-Performance Air-Stable $\mathrm{n}$-Type Transistors with an Asymmetrical Device Configuration Based on Organic Single-Crystalline Submicrometer/Nanometer Ribbons. J. Am. Chem. Soc. 2006, 128, 14634-14639.

(22) Asadi, K.; Wu, Y.; Gholamrezaie, F.; Rudolf, P.; Blom, P. W. M. Single-Layer Pentacene Field-Effect Transistors Using Electrodes Modified With Self-assembled Monolayers. Adv. Mater. 2009, 21, 4109-4114.

(23) Klauk, H.; Schmid, G.; Radlik, W.; Weber, W.; Zhou, L.; Sheraw, C. D.; Nichols, J. A.; Jackson, T. N. Contact resistance in organic thin film transistors. Solid-State Electron. 2003, 47, 297-301.

(24) Heimel, G.; Romaner, L.; Zojer, E.; Brédas, J.-L. The Interface Energetics of Self-Assembled Monolayers on Metals. Acc. Chem. Res. 2008, 41, 721-729.

(25) Zhu, X.; Yan, W.; Levy, U.; Mortensen, N. A.; Kristensen, A. Resonant laser printing of structural colors on high-index dielectric metasurfaces. Sci. Adv. 2017, 3, No. e1602487. 